九州大学学術情報リポジトリ

Kyushu University Institutional Repository

\title{
Notes on Estimating Inverse-Gaussian and Gamma Subordinators under High-frequency Sampling
}

Masuda, Hiroki

Graduate School of Mathematics, Kyushu University

http://hdl. handle. net/2324/3395

出版情報 : MHF Preprint Series. 2006-28，2006-09-14. 九州大学大学院数理学研究院 バージョン：

権利関係 : 


\title{
MHF Preprint Series
}

Kyushu University

21st Century COE Program

Development of Dynamic Mathematics with

High Functionality

\section{Notes on estimating inverse-Gaussian and gamma subordinators under high-frequency sampling}

\author{
H. Masuda
}

MHF 2006-28

( Received September 14, 2006 )

Faculty of Mathematics

Kyushu University

Fukuoka, JAPAN 


\title{
Notes on Estimating Inverse-Gaussian and Gamma Subordinators under High-frequency Sampling *
}

\author{
Hiroki Masuda ${ }^{\dagger}$ \\ Graduate School of Mathematics, Kyushu University, \\ 6-10-1 Hakozaki, Higashi-ku, Fukuoka 812-8581, Japan
}

Version: September 14, 2006

\begin{abstract}
We study joint efficient estimation of two parameters dominating gamma and inverseGaussian subordinators, based on discrete observations sampled at $\left(t_{i}^{n}\right)_{i=1}^{n}$ satisfying $h_{n}:=\max _{i \leq n}\left(t_{i}^{n}-t_{i-1}^{n}\right) \rightarrow 0$ as $n \rightarrow \infty$. Under the condition that $T_{n}:=t_{n}^{n} \rightarrow \infty$ as $n \rightarrow \infty$ we have two kinds of optimal rates, $\sqrt{n}$ and $\sqrt{T_{n}}$, and especially. Moreover, as in estimation of diffusion coefficient of a Wiener process the $\sqrt{n}$-consistent component of the estimator is effectively workable even when $T_{n}$ does not tend to infinity. Simulation experiments are given under several $h_{n}$ 's behaviors.
\end{abstract}

\section{Introduction}

In this article we shall present two case studies of estimating a subordinator based on a kind of high-frequency discrete data. A subordinator $Z=\left(Z_{t}\right)_{t \in \mathbb{R}_{+}}$is a one-dimensional nondecreasing càdlàg (right continuous and having left hand side limits) process a.s. starting from the origin with independent and stationary increments. For any subordinator without drift, there corresponds a Lévy measure $\nu$ satisfying $\int_{0}^{1}|z| \nu(d z)<\infty$ and supported by $\mathbb{R}_{+}$ for which

$$
\varphi_{Z_{t}}(u)=\exp \left\{t \int\left(e^{i u z}-1\right) \nu(d z)\right\}, \quad u \in \mathbb{R}, t \in \mathbb{R}_{+} .
$$

This is a special case of the so called Lévy-Khintchine formula. Here and in the sequel $u \mapsto \varphi_{\xi}(u)$ stands for the characteristic function of $\xi$, a random variable or a distribution. Given a subordinator $Z$ the law at time $1, \mathcal{L}\left(Z_{1}\right)$, is uniquely associated with an infinitely divisible distribution whose support is contained in $\mathbb{R}_{+}=[0, \infty)$. See, e.g., Bertoin [3, Chapter III] for a systematic account of subordinators.

We shall consider statistical inference for two specific subordinators, $\mathcal{L}\left(Z_{1}\right)=I G(\delta, \gamma)$ and $\Gamma(\delta, \gamma)$, admitting the density (w.r.t. the Lebesgue measure)

$$
\begin{aligned}
& p(x ; \delta, \gamma)=\frac{\delta e^{\delta \gamma}}{\sqrt{2 \pi}} x^{-3 / 2} \exp \left\{-\frac{1}{2}\left(\gamma^{2} x+\frac{\delta^{2}}{x}\right)\right\} \mathbf{1}_{\mathbb{R}_{+}}(x), \\
& p(x ; \delta, \gamma)=\frac{\gamma^{\delta}}{\Gamma(\delta)} x^{\delta-1} \exp (-\gamma x) \mathbf{1}_{\mathbb{R}_{+}}(x),
\end{aligned}
$$

${ }^{*}$ This work was partly supported by Grant-in-Aid for Scientific Research from the Ministry of Education, Japan, and by the 21st Century COE Program "Development of Dynamic Mathematics with High Functionality" at Kyushu University.

†email: hiroki@math.kyushu-u.ac.jp 
respectively, where $\delta$ and $\gamma$ are positive constants; both of the inverse-Gaussian and gamma subordinators have infinitely many jumps over each finite time interval as the Lévy measure $\nu$ in the formula (1) is given by $g(z ; \delta, \gamma)=\delta(2 \pi)^{-1 / 2} z^{-3 / 2} \exp \left(-\gamma^{2} z / 2\right) \mathbf{1}_{\mathbb{R}_{+}}(z)$ and $g(z ; \delta, \gamma)=$ $\delta z^{-1} \exp (-\gamma z) \mathbf{1}_{\mathbb{R}_{+}}(z)$, respectively.

We are interested in estimating $\theta=(\delta, \gamma)$ when available data is

$$
Z_{t_{0}^{n}}, Z_{t_{1}^{n}}, \ldots, Z_{t_{n}^{n}}
$$

where $\left(t_{i}^{n}\right)_{i=0}^{n}$ is a nonrandom positive sequence satisfying

$$
0 \equiv t_{0}^{n}<t_{1}^{n}<\cdots<t_{n}^{n}=: T_{n}
$$

for each $n \in \mathbb{N}$. Throughout this article we suppose

$$
\left\{\begin{array}{l}
h_{n}:=\max _{1 \leq i \leq n}\left(t_{i}^{n}-t_{i-1}^{n}\right) \rightarrow 0 \\
T_{n} \asymp n h_{n}
\end{array}\right.
$$

as $n \rightarrow \infty$, where $a_{n} \asymp b_{n}$ means that there exists a constant $c>0$ such that $c^{-1} \leq a_{n} / b_{n} \leq c$ for every $n$ large enough. For joint estimation of $\delta$ and $\gamma$ we shall additionally suppose $T_{n} \rightarrow \infty$; then the sampling scheme is asymptotically the same as in the case where $Z$ is continuously observed over $\left[0, T_{n}\right]$ with $T_{n} \rightarrow \infty$. If we can observe continuous data $\left(Z_{t}\right)_{t \in[0, T]}$, the likelihood theory has been already established: see, e.g., Akritas [1] and Akritas and Johnson [2]. Denote by $P_{\theta}^{T}$ the law of a sample path $\left(X_{t}\right)_{t \in[0, T]}$ on the Skorohod space (i.e., the space of càdlàg processes endowed with the Skorohod topology), and fix any $\theta^{i}=\left(\delta^{i}, \gamma^{i}\right), i=1,2$, and $T>0$. Then, in both of the inverse-Gaussian and gamma cases, $P_{\theta^{1}}^{T}$ and $P_{\theta^{2}}^{T}$ fail to be mutually absolutely continuous as soon as $\delta^{1} \neq \delta^{2}$ (see, e.g., Akritas and Johnson [2, Theorem 4.1]), so that we cannot consider the joint likelihood estimation of $\delta$ and $\gamma$ from a continuous record while it makes sense in discrete-observation cases. If $t_{i}^{n}-t_{i-1}^{n} \equiv h>0$ in particular, then the situation is nothing but the classical iid framework, and the convergence rate of the MLE is of course $\sqrt{n} I_{2}$ for both component, and we can explicitly write down the corresponding Fisher information matrices (depending on $h$ in this case): Woerner [9] systematically studied such cases for much more general classes of Lévy processes.

Our main goal is to derive uniform asymptotic behaviors of the corresponding maximumlikelihood estimators (MLE), say $\hat{\theta}_{n}=\left(\hat{\delta}_{n}, \hat{\gamma}_{n}\right)$. In both cases we presuppose:

that the parameter space $\Theta \subset(0, \infty)^{2}$ is a bounded domain whose closure is contained in $(0, \infty)^{2}$ and that there is a true parameter which lies in $\Theta$.

Denote by $P_{\theta}^{n}$ the image measure of $\left(Z_{t_{i}^{n}}\right)_{i=0}^{n}$ associated with $\theta$. We shall derive the local asymptotic normality (LAN) as well as the asymptotic normality of the MLEs with rate $\operatorname{diag}\left(\sqrt{n}, \sqrt{T_{n}}\right)$, both uniform in $\Theta$. Here the asymptotic normality with rate $\operatorname{diag}\left(\sqrt{n}, \sqrt{T_{n}}\right)$ means that the convergence in law

$$
\left(\sqrt{n}\left(\hat{\delta}_{n}-\delta\right), \sqrt{T_{n}}\left(\hat{\gamma}_{n}-\gamma\right)\right) \Rightarrow \mathcal{N}_{2}\left(0, I(\theta)^{-1}\right)
$$

under $\left(P_{\theta}^{n}\right)$-sequence of distributions, see (9) and (13) for specified expressions of $I(\theta)$ : this is a similar phenomenon to the well known case where $Z$ is a Wiener process such that $\mathcal{L}\left(Z_{1}\right)=\mathcal{N}_{1}(\gamma, \delta)$, or, more generally, a diffusion process. Under our sampling scheme (4), we shall see that a remarkably different feature from the case of $t_{i}^{n}-t_{i-1}^{n} \equiv h>0$ will arise. Precisely, the forms of the asymptotic Fisher information matrices are different, and especially they are diagonal, which implies that the joint ML estimation of $\delta$ and $\gamma$ is asymptotically mutually independent. See the expressions (9) and (13) below. We shall also derive an efficient estimator of $\delta$ even when $\left(T_{n}\right)$ is bounded in $n$; in this case $\gamma$ may be unknown, hence a nuisance parameter. 
Recall that, for general Lévy processes the likelihood function can be written down only up to the Fourier inversion formula. Jongbloed and van der Meulen [5] studied the parametric estimation of a subordinator based on the empirical characteristic function, where that $t_{i}^{n}-$ $t_{i-1}^{n} \equiv h>0$ is supposed and the efficiency issue is not discussed. Restricting the model structure, we shall give sharper results than theirs. As a matter of fact, specification of the parametric optimal rates in estimating a general Lévy process seems to be an intricate problem. For example, the author [6] previously studied the LAN property for discretely observed (non-Gaussian) stable Lévy processes, where various optimal rates were found for each component: there the scaling property, which is inherent in the stable case among general Lévy processes, was fully utilized, and it has been shown that the Fisher information matrix is always degenerate as long as joint estimation of scale and index parameters are concerned. Such a phenomenon does not arise in the present context.

In the rest of this article we shall present our asymptotic results in Section 2, then some simulation results in Section 3, and finally the proofs in Section 4.

\section{Results}

We use asymptotic symbols for $n \rightarrow \infty$ unless otherwise stated. Write $\Delta_{i}^{n}=t_{i}^{n}-t_{i-1}^{n}$, $\Delta_{i}^{n} Z=Z_{t_{i}^{n}}-Z_{t_{i-1}^{n}}, \theta:=(\delta, \gamma)$, and $\partial_{\theta}=\partial / \partial \theta$. Note that the sequence $\left(\Delta_{i}^{n} Z\right)_{i=1}^{n}$ forms a rowwise independent triangular array fulfilling

$$
\mathcal{L}\left(\Delta_{i}^{n} Z\right)=\mathcal{L}\left(Z_{\Delta_{i}^{n} t}\right)
$$

for each $i \in\{1,2, \ldots, n\}$. For any $\sigma\left(Z_{t_{i}^{n}}: i \leq n\right)$-measurable random variables $X_{n}(\theta), n \in \mathbb{N}$, and a constant $X(\theta)$ appearing in the sequel, we write:

(i) " $X_{n}(\theta) \Rightarrow_{u}^{P_{\theta}^{n}} X(\theta)$ " if

$$
\sup _{\theta \in \Theta^{-}}\left|P^{X_{n}(\theta)} f-P^{X(\theta)} f\right| \rightarrow 0
$$

for every bounded continuous function $f$, where $P^{\xi}$ denotes the law of $\xi$;

(ii) " $X_{n}(\theta) \rightarrow{ }_{u}^{P_{\theta}^{n}} X(\theta)$ " if for every $\epsilon>0$ we have

$$
\sup _{\theta \in \Theta^{-}} P_{\theta}^{n}\left[\left|X_{n}(\theta)-X(\theta)\right|>\epsilon\right] \rightarrow 0 .
$$

Given a $\log$-likelihood function $\theta \mapsto \ell_{n}(\theta)$, we write

$$
\mathcal{S}_{n}(\theta)=\partial_{\theta} \ell_{n}(\theta) \quad \text { and } \quad \mathcal{I}_{n}(\theta)=-\partial_{\theta}^{2} \ell_{n}(\theta)
$$

the score function and the observed information matrix, respectively.

With the above-mentioned notation, we formulate the uniform LAN property in our context as follows. Write

$$
A_{n}=\operatorname{diag}\left(\sqrt{n}, \sqrt{T_{n}}\right)
$$

so that $A_{n}^{-1} \rightarrow 0$. The experiment $\left\{P_{\theta}^{n}: n \in \mathbb{N}\right\}_{\theta \in \Theta}$ is called "uniformly $A_{n}$-LAN with the Fisher informations $\{I(\theta)\}_{\theta \in \Theta}$ " if:

[U1] $\ell_{n}\left(\theta+A_{n}^{-1 \top} u_{n}\right)-\ell_{n}(\theta)-u_{n}^{\top} \mathcal{S}_{n}(\theta)+\frac{1}{2} u_{n}^{\top} \mathcal{I}_{n}(\theta) u_{n} \rightarrow{ }_{u}^{P_{\theta}^{n}} 0$ for any nonrandom bounded sequence $\left(u_{n}\right) \subset \mathbb{R}^{2}$ such that $u_{n} \rightarrow u$;

[U2] $A_{n}^{-1} \mathcal{S}_{n}(\theta) \Rightarrow{ }_{u}^{n} \mathcal{N}_{2}(0, I(\theta))$ with $I(\theta)$ being positive definite for any $\theta \in \Theta^{-}$;

[U3] $A_{n}^{-1} \mathcal{I}_{n}(\theta) A_{n}^{-1 \top} \rightarrow{ }_{u}^{n}{ }_{\theta}^{n} I(\theta)$, with the same $I(\theta)$ as in [U2].

The forthcoming asymptotic normalities reveal that the MLEs are asymptotically efficient in both of inverse-Gaussian and gamma cases. If $\hat{\theta}_{n} \in \Theta^{-}$is not well-defined, we may assign any number $\theta \in \Theta$ to $\hat{\theta}_{n}$. 


\subsection{Inverse-Gaussian case}

Let $\mathcal{L}\left(Z_{1}\right)=I G(\delta, \gamma)$ whose density is given by (2). Since

$$
\varphi_{I G(\delta, \gamma)}(u)=\exp \left\{\delta\left(\gamma-\sqrt{\gamma^{2}-2 i u}\right)\right\}
$$

we have $\mathcal{L}\left(Z_{t}\right)=I G(\delta t, \gamma)$ for each $t>0$. On account of (5), the target log-likelihood function of $\left(Z_{t_{i}^{n}}\right)_{i=0}^{n}$ is given by

$$
\ell_{n}(\theta)=\sum_{i=1}^{n}\left\{\log \delta+\delta \gamma \Delta_{i}^{n} t-\frac{1}{2}\left(\frac{\delta^{2}\left(\Delta_{i}^{n} t\right)^{2}}{\Delta_{i}^{n} Z}+\gamma^{2} \Delta_{i}^{n} Z\right)\right\} .
$$

Solving $\partial_{\theta} \ell_{n}(\theta)=0$, we get the explicit MLE:

$$
\hat{\delta}_{n}=\left[\frac{1}{n}\left\{\sum_{i=1}^{n} \frac{\left(\Delta_{i}^{n} t\right)^{2}}{\Delta_{i}^{n} Z}-\frac{T_{n}^{2}}{Z_{T_{n}}}\right\}\right]^{-1 / 2}, \quad \hat{\gamma}_{n}=\frac{T_{n} \hat{\delta}_{n}}{Z_{T_{n}}} .
$$

For the joint estimation we have the following.

Theorem 2.1 (Unbounded-domain asymptotics). Let $Z$ be a subordinator such that $\mathcal{L}\left(Z_{1}\right)=$ $I G(\delta, \gamma)$, let $\ell_{n}(\theta)$ and $\hat{\theta}_{n}=\left(\hat{\delta}_{n}, \hat{\gamma}_{n}\right)$ be as in (7) and (8), respectively, and suppose (4) and $T_{n} \rightarrow \infty$. Then $\left\{P_{\theta}^{n}: n \in \mathbb{N}\right\}_{\theta \in \Theta}$ is uniformly $A_{n}-L A N$ with the Fisher informations

$$
I_{I G}(\theta)=\left(\begin{array}{cc}
2 / \delta^{2} & 0 \\
0 & \delta / \gamma
\end{array}\right), \quad \theta \in \Theta
$$

and we have $A_{n}\left(\hat{\theta}_{n}-\theta\right) \Rightarrow_{u}^{P_{\theta}^{n}} \mathcal{N}_{2}\left(0, I_{I G}(\theta)^{-1}\right)$.

If $T_{n}$ does not tends to infinity, then the observed information associated with $\gamma$ is bounded in $n$, and this is the case also for the gamma Lévy process; see (16) and (17). Therefore no consistent estimation procedure of $\gamma$ is possible. But this is not the case for estimating $\delta$, and actually we may use the same estimate as in (8).

Corollary 2.2 (Bounded-domain asymptotics). Let $Z$ be a subordinator such that $\mathcal{L}\left(Z_{1}\right)=$ $I G(\delta, \gamma)$, where $\gamma>0$ is fixed while it may be unknown, let $\hat{\delta}_{n}$ be given by (8), and suppose (4) and $T_{n}=O(1)$. Moreover suppose that the true value of $\delta$ lies in some interval $(a, b)$ such that $0<a<b<\infty$. Then $\left\{P_{\delta}^{n}: n \in \mathbb{N}\right\}_{\delta \in(a, b)}$ is uniformly $\sqrt{n}-L A N$ with the Fisher informations $2 / \delta^{2}$, and $\hat{\delta}_{n}$ fulfils $\sqrt{n}\left(\hat{\delta}_{n}-\delta\right) \Rightarrow{ }_{u}^{P_{\delta}^{n}} \mathcal{N}_{1}\left(0, \delta^{2} / 2\right)$.

\subsection{Gamma case}

Next we set $\mathcal{L}\left(Z_{1}\right)=\Gamma(\delta, \gamma)$ whose density is given by $(3)$, so that

$$
\varphi_{\Gamma(\delta, \gamma)}(u)=(1-i u / \gamma)^{-\delta}
$$

and hence $\mathcal{L}\left(Z_{t}\right)=\Gamma(\delta t, \gamma)$ for each $t>0$. Thus the log-likelihood function of $\left(Z_{t_{i}^{n}}\right)_{i=0}^{n}$ is given by

$$
\ell_{n}(\theta)=\sum_{i=1}^{n}\left\{\delta \Delta_{i}^{n} t \log \gamma-\log \Gamma\left(\delta \Delta_{i}^{n} t\right)+\delta \Delta_{i}^{n} t \log \left(\Delta_{i}^{n} Z\right)-\gamma \Delta_{i}^{n} Z\right\} .
$$

The corresponding MLE solves

$$
\begin{aligned}
\sum_{i=1}^{n}\left(\Delta_{i}^{n} t\right)\left\{\log \left(\delta \Delta_{i}^{n} t\right)-\psi\left(\delta \Delta_{i}^{n} t\right)\right\} & =T_{n} \log \left(\frac{Z_{T_{n}}}{T_{n}}\right)-\sum_{i=1}^{n}\left(\Delta_{i}^{n} t\right) \log \left(\frac{\Delta_{i}^{n} Z}{\Delta_{i}^{n} t}\right), \\
\gamma & =\delta \frac{T_{n}}{Z_{T_{n}}},
\end{aligned}
$$




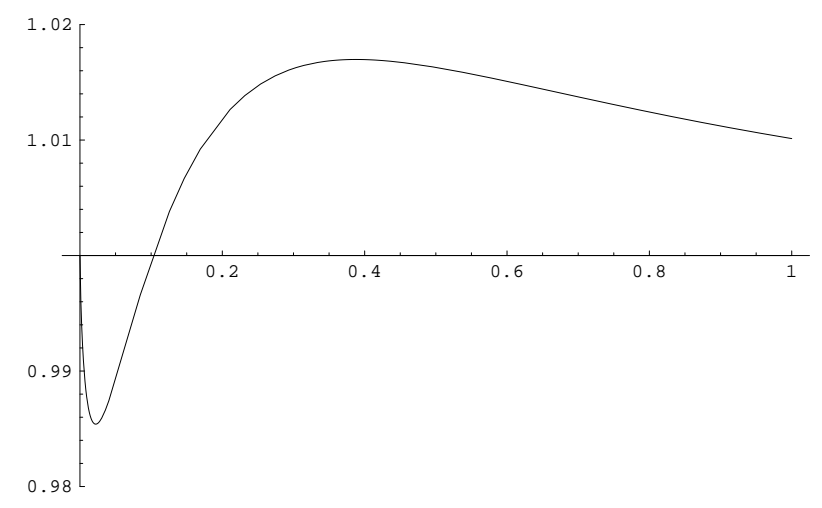

Figure 1: The plot of the bounded function $(0, \infty) \ni x \mapsto\{\log x-\psi(x)\} /\left\{(3 x+1) /\left(6 x^{2}+x\right)\right\}$, which is strictly increasing to 1 (resp. decreasing to 0 ) as $x \searrow 0$ (resp. as $x \nearrow \infty$ ).

where $\psi$ denotes the digamma function, $\psi(x):=\partial_{x} \Gamma(x) / \Gamma(x)$.

For each $n \in \mathbb{N}$ the left-hand side of $(12)$, say $f_{n}(\delta)$, is a smooth, positive, and strictly decreasing function of $\delta \in(0, \infty): f_{n}(x) \searrow 0(\operatorname{resp} . \nearrow \infty)$ as $x \nearrow \infty(\operatorname{resp} . x \searrow 0)$. So (11) admits a unique root $\hat{\delta}_{n}$ a.s. on the event where the right-hand side of (11) is positive, and we can simply apply, e.g., the bisection search in order to find the root of (11) readily.

Theorem 2.3 (Unbounded-domain asymptotics). Let $Z$ be a subordinator such that $\mathcal{L}\left(Z_{1}\right)=$ $\Gamma(\delta, \gamma)$, and let $\ell_{n}(\theta)$ and $\hat{\theta}_{n}=\left(\hat{\delta}_{n}, \hat{\gamma}_{n}\right)$ be as in (10) and the solution to (11) and (12), respectively, and suppose (4) and $T_{n} \rightarrow \infty$. Then $\left\{P_{\theta}^{n}: n \in \mathbb{N}\right\}_{\theta \in \Theta}$ is uniformly $A_{n}-L A N$ with the Fisher informations

$$
I_{\Gamma}(\theta)=\left(\begin{array}{cc}
1 / \delta^{2} & 0 \\
0 & \delta / \gamma^{2}
\end{array}\right), \quad \theta \in \Theta
$$

and we have $A_{n}\left(\hat{\theta}_{n}-\theta\right) \Rightarrow{ }_{u}^{P_{\theta}^{n}} \mathcal{N}_{2}\left(0, I_{\Gamma}(\theta)^{-1}\right)$.

We also have an analogue to Corollary 2.2.

Corollary 2.4 (Bounded-domain asymptotics). Let $Z$ be a subordinator such that $\mathcal{L}\left(Z_{1}\right)=$ $\Gamma(\delta, \gamma)$, where $\gamma>0$ is fixed while it may be unknown, let $\hat{\delta}_{n}$ be a solution of (11), and suppose (4) and $T_{n}=O(1)$. Moreover, suppose that the true value of $\delta$ lies in $(a, b)$ for some $0<a<b<\infty$. Then $\left\{P_{\delta}^{n}: n \in \mathbb{N}\right\}_{\delta \in(a, b)}$ is uniformly $\sqrt{n}$-LAN with the Fisher informations $1 / \delta^{2}$, and $\hat{\delta}_{n}$ fulfils $\hat{\delta}_{n}$ fulfils $\sqrt{n}\left(\hat{\delta}_{n}-\delta\right) \Rightarrow{ }_{u}^{P_{\theta}^{n}} \mathcal{N}_{1}\left(0, \delta^{2}\right)$.

Here is a remark for finding the root of (11) in the equidistant-sampling case, $h_{n}=$ $t_{i}^{n}-t_{i-1}^{n}$ for each $n$ : in this case (11) can be rewritten as

$$
\log \left(\delta h_{n}\right)-\psi\left(\delta h_{n}\right)=\log \left(\frac{1}{n} \sum_{i=1}^{n} \Delta_{i}^{n} Z\right)-\frac{1}{n} \sum_{i=1}^{n} \log \left(\Delta_{i}^{n} Z\right) .
$$

Write this right-hand side as $Y_{n}$. We can show that $h_{n} Y_{n} \rightarrow{ }_{u}^{P_{\theta}^{n}} \delta^{-1}>0$ (use Genon-Catalot and Jacod [4, Lemma 9]), hence $Y_{n}$ becomes positive with $P_{\theta}^{n}$-probability tending to 1 . Now, using the approximation (see Figure 1)

$$
\log (x)-\psi(x) \sim \frac{3 x+1}{6 x^{2}+x}, \quad x \rightarrow 0,
$$

and taking the positivity of $\delta$ into account, we get the approximate MLE $\tilde{\delta}_{n}$ of $\delta$ given by

$$
\tilde{\delta}_{n}=\frac{3-Y_{n}}{12 h_{n} Y_{n}}+\left\{\left(\frac{3-Y_{n}}{12 h_{n} Y_{n}}\right)^{2}+\frac{1}{6 h_{n} Y_{n}}\right\}^{1 / 2},
$$




\begin{tabular}{rrrrrr}
\hline$n$ & $T_{n}$ & $\hat{\delta}_{n}^{I G}$-mean (s.d.) & $\hat{\gamma}_{n}^{I G}$-mean (s.d.) & $\tilde{\delta}_{n}^{\Gamma}$-mean (s.d.) & $\tilde{\gamma}_{n}^{\Gamma}$-mean (s.d.) \\
\hline 50 & 3.23 & $3.0910(0.3195)$ & $2.1514(0.5217)$ & $3.0674(0.4832)$ & $2.2386(0.8505)$ \\
100 & 3.98 & $3.0276(0.2243)$ & $2.0865(0.4657)$ & $3.0604(0.3249)$ & $2.2331(0.7651)$ \\
300 & 5.54 & $3.0142(0.1233)$ & $2.0560(0.3547)$ & $3.0499(0.1798)$ & $2.1650(0.6089)$ \\
500 & 6.45 & $3.0021(0.0929)$ & $2.0657(0.3410)$ & $3.0426(0.1321)$ & $2.1241(0.5064)$ \\
\hline
\end{tabular}

Table 1: Simulation 1. Means and standard deviations (s.d.) of the estimate based on 1000 independent trajectories. We set $h_{n}=n^{-0.7}$, i.e. $T_{n}=n^{0.3} \rightarrow \infty$, and $(\delta, \gamma)=(3,2)$.

which, together with $\tilde{\gamma}_{n}:=\tilde{\delta}_{n} T_{n} / Z_{T_{n}}$ in cases of $T_{n} \rightarrow \infty$, enables us to bypass the numerical optimization procedure.

Remark 2.5. As a familiar naive estimator, one may consider moment estimator based on the first and second sample moments, utilizing the convergences

$$
\frac{1}{T_{n}} \sum_{i=1}^{n} \Delta_{i}^{n} Z \rightarrow P_{\theta}^{n} \frac{\delta}{\gamma} \text { and } \frac{1}{T_{n}} \sum_{i=1}^{n}\left(\Delta_{i}^{n} Z\right)^{2} \rightarrow P_{\theta}^{n} \frac{\delta}{\gamma^{2}} ;
$$

again, this can be proved by means of [4, Lemma 9]. However, the asymptotic behavior of the moment estimator $\hat{\theta}_{M, n}$ obtained from the above relations are far from that of $\hat{\theta}_{n}$ : using the delta method we can check

$$
\sqrt{T_{n}}\left(\hat{\theta}_{M, n}-\theta\right) \Rightarrow \mathcal{N}_{2}\left(0,\left(\begin{array}{cc}
2 \delta & 2 \gamma \\
2 \gamma & 3 \gamma^{2} / \delta
\end{array}\right)\right)
$$

This reveals that we cannot use $\hat{\theta}_{M, n}$ even as an initial estimate in applying Fisher's scoring or one-step improvement.

\section{Simulation experiments}

We here report some numerical results. For simplicity we carried out equidistant sampling cases, $\Delta_{i}^{n} t=h_{n}$ for each $i \leq n$. For gamma cases, we utilized the approximate MLE $\tilde{\theta}_{n}:=\left(\tilde{\delta}_{n}, \tilde{\gamma}_{n}\right)$; the results will show that even $\tilde{\theta}_{n}$ performs well. In each simulation we simulated 1000 independent discrete sample paths of $Z$, and then computed mean and standard deviation (s.d.) of the obtained 1000 estimates. Throughout the true value is $(\delta, \gamma)=(3,2)$. For generating pseudorandom- $\Gamma(p, q)$ numbers with $p \in(0,1)$, we used the algorithm of Michael et al. [7].

In Tables 1 and 2, we distinguish inverse-Gaussian and gamma cases by the superscripts " $I G$ " and " $\Gamma "$ ".

Simulation 1. Next we set $h_{n}=n^{-0.7}$, so that $T_{n}=n^{0.3} \rightarrow \infty$ and the jointly consistent estimation of $\delta$ and $\gamma$ can be done. The results are given in Table 1.

Simulation 2. Next we set $h_{n}=n^{-0.3}$, so that $T_{n}=n^{0.7} \rightarrow \infty$; the total observation-time domain diverges faster than the previous case. It is observed that: (i) accuracy of estimating $\delta$ is slightly worse than Simulation 1; and (ii) performance of estimating $\gamma$ is much better than Simulation 1, because of the faster increase of $T_{n}$. The results are given in Table 2 .

Finally, let us look at a case of $T_{n}=O(1)$ in the inverse-Gaussian case.

Simulation 3. Set $h_{n}=1 / n$, so that $T_{n} \equiv 1$. In this case only $\delta$ can be consistently estimated. The results are given in Table 3: just for reference we also give estimates $\hat{\gamma}_{n}$, which badly behaved and have severe inevitable bias, as was expected. 


\begin{tabular}{rrlrrr}
\hline$n$ & $T_{n}$ & $\hat{\delta}_{n}^{I G}$-mean (s.d) & $\hat{\gamma}_{n}^{I G}$-mean (s.d.) & $\tilde{\delta}_{n}^{\Gamma}$-mean (s.d) & $\tilde{\gamma}_{n}^{\Gamma}$-mean (s.d.) \\
\hline 50 & 15.46 & $3.0812(0.3190)$ & $2.0630(0.2941)$ & $3.1331(0.5908)$ & $2.1335(0.5235)$ \\
100 & 25.12 & $3.0384(0.2152)$ & $2.0487(0.2248)$ & $3.0319(0.3988)$ & $2.0470(0.3557)$ \\
300 & 54.20 & $3.0058(0.1224)$ & $2.0095(0.1392)$ & $2.9866(0.2059)$ & $2.0030(0.2130)$ \\
500 & 77.50 & $3.0115(0.0933)$ & $2.0087(0.1138)$ & $2.9713(0.1534)$ & $1.9911(0.1741)$ \\
\hline
\end{tabular}

Table 2: Simulation 2. Means and standard deviations (s.d.) of the estimate based on 1000 independent trajectories. We set $h_{n}=n^{-0.3}$, i.e. $T_{n}=n^{0.7} \rightarrow \infty,(\delta, \gamma)=(3,2)$.

\begin{tabular}{rcc}
\hline$n$ & $\hat{\delta}_{n}^{I G}$-mean (s.d) & $\hat{\gamma}_{n}^{I G}$-mean (s.d.) \\
\hline 50 & $3.0760(0.3220)$ & $2.3424(0.9575)$ \\
100 & $3.0431(0.2200)$ & $2.4193(0.9441)$ \\
300 & $3.0211(0.1230)$ & $2.3300(0.9481)$ \\
500 & $3.0114(0.0935)$ & $2.3123(0.9044)$ \\
1000 & $3.0011(0.0674)$ & $2.3603(0.9735)$ \\
\hline
\end{tabular}

Table 3: Simulation 3. Means and standard deviations (s.d.) of the estimate based on 1000 independent trajectories. We set $h_{n}=1 / n$, i.e. $T_{n} \equiv 1$.

\section{Proofs}

\subsection{Preliminary}

Put $H_{n}(\theta)=\left[H_{n}^{k l}(\theta)\right]_{k, l=1}^{n}=A_{n}^{-1} \mathcal{I}_{n}(\theta) A_{n}^{-1 \top}$, the normalized observed information matrix; recall (6). First, utilizing the results of Sweeting [8, Theorems 1 and 2], we shall observe that each proof of Theorems 2.1 and 2.3 reduces to the verification of [U3].

Put $\mathcal{I}_{n}(\theta)=\left[\mathcal{I}_{n}^{k l}(\theta)\right]_{k, l=1}^{2}$. Given $\theta^{k}=\left(\delta^{k}, \gamma^{k}\right), k=1,2$, we introduce the notation

$$
\mathcal{I}_{n}\left(\theta^{1}, \theta^{2}\right):=\left[\mathcal{I}_{n}^{k l}\left(\theta^{k}\right)\right]_{k, l=1}^{2},
$$

and, for a constant $a>0$,

$$
F_{n}^{a}(\theta)=\sup _{\theta^{k}:\left|A_{n}\left(\theta^{k}-\theta\right)\right| \leq a, k=1,2}\left|A_{n}^{-1}\left\{\mathcal{I}_{n}\left(\theta^{1}, \theta^{2}\right)-\mathcal{I}_{n}(\theta)\right\} A_{n}^{-1}\right|:
$$

these quantities appear in the assumptions of [8] in relation to deriving asymptotic results through Taylor's formula. Now suppose

$$
H_{n}(\theta) \rightarrow{ }_{\theta}^{n} H(\theta)
$$

for some $H(\theta)$, which is positive definite for every $\theta \in \Theta^{-}$and continuous in $\theta \in \Theta$. Then we can easily see as follows that the condition C2(ii) of [8] is fulfilled. Write $A_{n}=\operatorname{diag}\left(A_{1 n}, A_{2 n}\right)$ and observe that for each $\theta^{k} \in \Theta$ we have

$$
\begin{aligned}
\mid A_{n}^{-1} & \left\{\mathcal{I}_{n}\left(\theta^{1}, \theta^{2}\right)-\mathcal{I}_{n}(\theta)\right\} A_{n}^{-1} \mid \\
& \lesssim \sum_{k, l=1}^{2}\left|A_{k n}^{-1} A_{l n}^{-1}\left\{\mathcal{I}_{n}^{k l}\left(\theta^{k}\right)-\mathcal{I}_{n}^{k l}(\theta)\right\}\right| \\
& \left.\leq \sum_{k, l=1}^{2}\left\{\left|H_{n}^{k l}\left(\theta^{k}\right)-H^{k l}\left(\theta^{k}\right)\right|+\mid H_{n}^{k l}(\theta)-H^{k l}(\theta)\right\}|+| H^{k l}\left(\theta^{k}\right)-H^{k l}(\theta) \mid\right\},
\end{aligned}
$$

and therefore, on account of (15), we get $F_{n}^{a}(\theta) \rightarrow{ }_{u}^{P_{\theta}^{n}} 0$ for every $a>0$ as desired. In particular, this leads to [U1]. Since the conditions C1 and C2(i) of [8] are automatic under (15), 
Theorems 1 and 2 of the paper can apply under (15), so that we get [U2], the existence of a local root of $\partial_{\theta} \ell_{n}(\theta)=0$, and the uniform asymptotic normality $\left\{A_{n}^{-1} \mathcal{I}_{n}(\theta) A_{n}^{-1 \top}\right\}^{1 / 2} A_{n}\left(\hat{\theta}_{n}-\right.$ $\theta) \Rightarrow{ }_{\theta}^{P_{\theta}^{n}} \mathcal{N}_{2}\left(0, I_{2}\right)$, where $I_{2}$ denotes the 2-dimensional identity matrix.

Thus, in order to prove Theorem 2.1 (resp. Theorem 2.3), it suffices to show (15), namely $H_{n}(\theta) \rightarrow{ }_{u}^{P_{\theta}^{n}} I_{I G}(\theta)\left(\right.$ resp. $\left.H_{n}(\theta) \rightarrow{ }_{u}^{P_{\theta}^{n}} I_{\Gamma}(\theta)\right)$.

\subsection{Inverse-Gaussian case}

Proof of Theorem 2.1. Direct computations yield

$$
H_{n}(\theta)=\left(\begin{array}{cc}
\frac{1}{\delta^{2}}+\frac{1}{n} \sum_{i=1}^{n} \frac{\left(\Delta_{i}^{n} t\right)^{2}}{\Delta_{i}^{n} Z} & -\sqrt{\frac{T_{n}}{n}} \\
\operatorname{sym} . & \frac{1}{T_{n}} \sum_{i=1}^{n} \Delta_{i}^{n} Z
\end{array}\right) .
$$

The task here is to show $H_{n}(\theta) \rightarrow_{u}^{P_{\theta}^{n}} I_{I G}(\theta)$ (recall (9)), but in view of (4) it is clear that $H_{n}^{12}(\theta)=H_{n}^{21}(\theta) \rightarrow_{u} 0$ (the ordinary convergence). As for the diagonal elements, we shall first utilize [4, Lemma 9] to deduce the $\theta$-pointwise convergence. Fix any $\theta \in \Theta$ and observe that

$$
\begin{aligned}
E_{\theta}^{n}\left[\Delta_{i}^{n} Z\right] & =\delta \Delta_{i}^{n} t / \gamma, \\
E_{\theta}^{n}\left[\left(\Delta_{i}^{n} Z\right)^{2}\right] & =\delta \Delta_{i}^{n} t / \gamma^{3}+\left(\delta \Delta_{i}^{n} t / \gamma\right)^{2}, \\
E_{\theta}^{n}\left[\left(\Delta_{i}^{n} Z\right)^{-1}\right] & =1 /\left\{\delta \Delta_{i}^{n} t\right\}^{2}+\gamma /\left(\delta \Delta_{i}^{n} t\right), \\
E_{\theta}^{n}\left[\left(\Delta_{i}^{n} Z\right)^{-2}\right] & =1 /\left\{\delta \Delta_{i}^{n} t\right\}^{4}+3 \gamma /\left\{\delta \Delta_{i}^{n} t\right\}^{3}+\left(2+\gamma^{2}\right) /\left\{\delta \Delta_{i}^{n} t\right\}^{2} .
\end{aligned}
$$

Now consider $H_{n}^{11}(\theta)$. Under (4) we have

$$
\begin{aligned}
\frac{1}{n} \sum_{i=1}^{n}\left(\Delta_{i}^{n} t\right)^{2} E_{\theta}^{n}\left[\left(\Delta_{i}^{n} Z\right)^{-1}\right] & =\frac{1}{\delta^{2}}+\frac{\gamma}{\delta} \frac{1}{n} \sum_{i=1}^{n}\left(\Delta_{i}^{n} t\right)^{2}=\frac{1}{\delta^{2}}+o(1), \\
\frac{1}{n^{2}} \sum_{i=1}^{n}\left(\Delta_{i}^{n} t\right)^{4} E_{\theta}^{n}\left[\left(\Delta_{i}^{n} Z\right)^{-2}\right] & =\frac{1}{n^{2}} \sum_{i=1}^{n} O(1)=o(1) .
\end{aligned}
$$

Thus [4, Lemma 9] yields $H_{n}^{11}(\theta) \rightarrow_{\theta}^{P^{n}} 2 / \delta^{2}$. Similarly, as for $H_{n}^{22}(\theta)$ we see

$$
\begin{aligned}
\frac{1}{T_{n}} \sum_{i=1}^{n} E_{\theta}^{n}\left[\Delta_{i}^{n} Z\right] & =\delta / \gamma, \\
\frac{1}{T_{n}^{2}} \sum_{i=1}^{n} E_{\theta}^{n}\left[\left(\Delta_{i}^{n} Z\right)^{2}\right] & =\frac{1}{T_{n}^{2}} \sum_{i=1}^{n}\left\{O(1)\left(\Delta_{i}^{n} t\right)^{2}+O(1) \Delta_{i}^{n} t\right\}=o(1),
\end{aligned}
$$

so that $H_{n}^{22}(\theta) \rightarrow_{\theta}^{P_{\theta}^{n}} \delta / \gamma$. The uniformity of the convergence of $H_{n}(\theta)$ directly follows on account of the continuity of $I_{I G}(\theta)$ and the boundedness of $\Theta$. Thus we obtain $H_{n}(\theta) \rightarrow{ }_{u}^{P_{\theta}^{n}}$ $I_{I G}(\theta)$ as desired.

Proof of Corollary 2.2. Note that the condition $T_{n} \rightarrow \infty$ was not used for $H_{n}^{11}(\theta)$ in the proof of Theorem 2.1. Hence the claim directly follows from Theorem 2.1 and the continuous mapping theorem. 


\subsection{Gamma case}

Proof of Theorem 2.3. We proceed along with the inverse-Gaussian case. We see that the observed information is nonrandom and $H_{n}(\theta)$ is given by

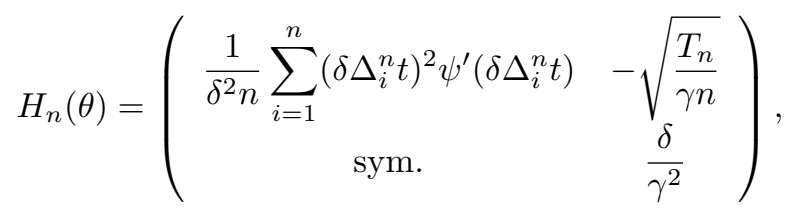

where $\psi^{\prime}$ denotes the derivative of $\psi$. It suffices to consider $H_{n}^{11}(\theta)$. We note that $x^{2} \psi^{\prime}(x) \rightarrow$ 1 as $x \searrow 0$. Put $C=\sup _{\delta} \delta^{-2}$, which is finite by means of the assumptions on $\Theta^{-}$. Given any $\epsilon>0$, from the boundedness of $\left(\Delta_{i}^{n} t\right)_{i=1}^{n}$ we can find $n_{0} \in \mathbb{N}$ such that

$$
\sup _{\theta \in \Theta} \sup _{n \geq n_{0}} \sup _{i \leq n}\left|\left(\delta \Delta_{i}^{n} t\right)^{2} \psi^{\prime}\left(\delta \Delta_{i}^{n} t\right)-1\right| \leq \epsilon / C .
$$

Hence for every $n \geq n_{0}$ we have

$$
\sup _{\theta \in \Theta}\left|H_{n}^{11}(\theta)-\delta^{-2}\right| \leq \sup _{\delta}\left\{\delta^{-2} \frac{1}{n} \sum_{i=1}^{n}\left|\left(\delta \Delta_{i}^{n} t\right)^{2} \psi^{\prime}\left(\delta \Delta_{i}^{n} t\right)-1\right|\right\} \leq C \cdot \epsilon / C=\epsilon,
$$

yielding that $H_{n}^{11}(\theta) \rightarrow{ }_{u} 1 / \delta^{2}$. This complete the proof.

Proof of Corollary 2.4. This follows every bit as Corollary 2.2.

\section{References}

[1] Akritas, M. G. (1982), Asymptotic theory for estimating the parameters of a Lévy process. Ann. Inst. Statist. Math. 34, 259-280.

[2] Akritas, M. G. and Johnson, R. A. (1981), Asymptotic inference in Lévy processes of the discontinuous type. Ann. Statist. 9, 604-614.

[3] Bertoin, J. (1996), Lévy Processes. Cambridge University Press, Cambridge.

[4] Genon-Catalot, V. and Jacod, J. (1993), On the estimation of the diffusion coefficient for multi-dimensional diffusion processes. Ann. Inst. H. Poincare Probab. Statist. 29, 119-151.

[5] Jongbloed, G. and van der Meulen, F. H. (2006), Parametric Estimation for Subordinators and Induced OU Processes, To appear in Scand. J. Statist.

[6] Masuda, H. (2006), Likelihood estimation of stable Lévy processes from discrete data. MHF preprint series 2006-18, Kyushu Univeresity.

[7] Michael, J. R., Schucany, W. R. and Haas, R. W. (1976), Generating random variates using transformations with multiple roots. Amer. Statist. 30, 88-90.

[8] Sweeting, T. J. (1980), Uniform asymptotic normality of the maximum likelihood estimator. Ann. Statist. 8, 1375-1381. [Corrections: (1982) Ann. Statist. 10, 320.]

[9] Woerner, J. H. C. (2001), Statistical Analysis for Discretely Observed Lévy Processes. PhD thesis, University of Freiburg. 


\section{List of MHF Preprint Series, Kyushu University 21st Century COE Program \\ Development of Dynamic Mathematics with High Functionality}

\section{MHF2005-1 Hideki KOSAKI}

Matrix trace inequalities related to uncertainty principle

MHF2005-2 Masahisa TABATA

Discrepancy between theory and real computation on the stability of some finite element schemes

MHF2005-3 Yuko ARAKI \& Sadanori KONISHI

Functional regression modeling via regularized basis expansions and model selection

MHF2005-4 Yuko ARAKI \& Sadanori KONISHI

Functional discriminant analysis via regularized basis expansions

MHF2005-5 Kenji KAJIWARA, Tetsu MASUDA, Masatoshi NOUMI, Yasuhiro OHTA \& Yasuhiko YAMADA

Point configurations, Cremona transformations and the elliptic difference Painlevé equations

MHF2005-6 Kenji KAJIWARA, Tetsu MASUDA, Masatoshi NOUMI, Yasuhiro OHTA \& Yasuhiko YAMADA

Construction of hypergeometric solutions to the $q$ - Painlevé equations

MHF2005-7 Hiroki MASUDA

Simple estimators for non-linear Markovian trend from sampled data:

I. ergodic cases

MHF2005-8 Hiroki MASUDA \& Nakahiro YOSHIDA

Edgeworth expansion for a class of Ornstein-Uhlenbeck-based models

MHF2005-9 Masayuki UCHIDA

Approximate martingale estimating functions under small perturbations of dynamical systems

MHF2005-10 Ryo MATSUZAKI \& Masayuki UCHIDA

One-step estimators for diffusion processes with small dispersion parameters from discrete observations

MHF2005-11 Junichi MATSUKUBO, Ryo MATSUZAKI \& Masayuki UCHIDA

Estimation for a discretely observed small diffusion process with a linear drift

MHF2005-12 Masayuki UCHIDA \& Nakahiro YOSHIDA

AIC for ergodic diffusion processes from discrete observations 
MHF2005-13 Hiromichi GOTO \& Kenji KAJIWARA

Generating function related to the Okamoto polynomials for the Painlevé IV equation

MHF2005-14 Masato KIMURA \& Shin-ichi NAGATA

Precise asymptotic behaviour of the first eigenvalue of Sturm-Liouville problems with large drift

MHF2005-15 Daisuke TAGAMI \& Masahisa TABATA

Numerical computations of a melting glass convection in the furnace

MHF2005-16 Raimundas VIDŪNAS

Normalized Leonard pairs and Askey-Wilson relations

MHF2005-17 Raimundas VIDŪNAS

Askey-Wilson relations and Leonard pairs

MHF2005-18 Kenji KAJIWARA \& Atsushi MUKAIHIRA

Soliton solutions for the non-autonomous discrete-time Toda lattice equation

MHF2005-19 Yuu HARIYA

Construction of Gibbs measures for 1-dimensional continuum fields

MHF2005-20 Yuu HARIYA

Integration by parts formulae for the Wiener measure restricted to subsets in $\mathbb{R}^{d}$

MHF2005-21 Yuu HARIYA

A time-change approach to Kotani's extension of Yor's formula

MHF2005-22 Tadahisa FUNAKI, Yuu HARIYA \& Mark YOR

Wiener integrals for centered powers of Bessel processes, I

MHF2005-23 Masahisa TABATA \& Satoshi KAIZU

Finite element schemes for two-fluids flow problems

MHF2005-24 Ken-ichi MARUNO \& Yasuhiro OHTA

Determinant form of dark soliton solutions of the discrete nonlinear Schrödinger equation

MHF2005-25 Alexander V. KITAEV \& Raimundas VIDŪNAS

Quadratic transformations of the sixth Painlevé equation

MHF2005-26 Toru FUJII \& Sadanori KONISHI

Nonlinear regression modeling via regularized wavelets and smoothing parameter selection

MHF2005-27 Shuichi INOKUCHI, Kazumasa HONDA, Hyen Yeal LEE, Tatsuro SATO, Yoshihiro MIZOGUCHI \& Yasuo KAWAHARA

On reversible cellular automata with finite cell array 
MHF2005-28 Toru KOMATSU

Cyclic cubic field with explicit Artin symbols

MHF2005-29 Mitsuhiro T. NAKAO, Kouji HASHIMOTO \& Kaori NAGATOU

A computational approach to constructive a priori and a posteriori error estimates for finite element approximations of bi-harmonic problems

MHF2005-30 Kaori NAGATOU, Kouji HASHIMOTO \& Mitsuhiro T. NAKAO Numerical verification of stationary solutions for Navier-Stokes problems

MHF2005-31 Hidefumi KAWASAKI

A duality theorem for a three-phase partition problem

MHF2005-32 Hidefumi KAWASAKI

A duality theorem based on triangles separating three convex sets

MHF2005-33 Takeaki FUCHIKAMI \& Hidefumi KAWASAKI

An explicit formula of the Shapley value for a cooperative game induced from the conjugate point

MHF2005-34 Hideki MURAKAWA

A regularization of a reaction-diffusion system approximation to the two-phase Stefan problem

MHF2006-1 Masahisa TABATA

Numerical simulation of Rayleigh-Taylor problems by an energy-stable finite element scheme

MHF2006-2 Ken-ichi MARUNO \& G R W QUISPEL

Construction of integrals of higher-order mappings

MHF2006-3 Setsuo TANIGUCHI

On the Jacobi field approach to stochastic oscillatory integrals with quadratic phase function

MHF2006-4 Kouji HASHIMOTO, Kaori NAGATOU \& Mitsuhiro T. NAKAO

A computational approach to constructive a priori error estimate for finite element approximations of bi-harmonic problems in nonconvex polygonal domains

MHF2006-5 Hidefumi KAWASAKI

A duality theory based on triangular cylinders separating three convex sets in $R^{n}$

MHF2006-6 Raimundas VIDŪNAS

Uniform convergence of hypergeometric series

MHF2006-7 Yuji KODAMA \& Ken-ichi MARUNO

N-Soliton solutions to the DKP equation and Weyl group actions 
MHF2006-8 Toru KOMATSU

Potentially generic polynomial

MHF2006-9 Toru KOMATSU

Generic sextic polynomial related to the subfield problem of a cubic polynomial

MHF2006-10 Shu TEZUKA \& Anargyros PAPAGEORGIOU

Exact cubature for a class of functions of maximum effective dimension

MHF2006-11 Shu TEZUKA

On high-discrepancy sequences

MHF2006-12 Raimundas VIDŪNAS

Detecting persistent regimes in the North Atlantic Oscillation time series

MHF2006-13 Toru KOMATSU

Tamely Eisenstein field with prime power discriminant

MHF2006-14 Nalini JOSHI, Kenji KAJIWARA \& Marta MAZZOCCO

Generating function associated with the Hankel determinant formula for the solutions of the Painlevé IV equation

MHF2006-15 Raimundas VIDŪNAS

Darboux evaluations of algebraic Gauss hypergeometric functions

MHF2006-16 Masato KIMURA \& Isao WAKANO

New mathematical approach to the energy release rate in crack extension

MHF2006-17 Toru KOMATSU

Arithmetic of the splitting field of Alexander polynomial

MHF2006-18 Hiroki MASUDA

Likelihood estimation of stable Lévy processes from discrete data

MHF2006-19 Hiroshi KAWABI \& Michael RÖCKNER

Essential self-adjointness of Dirichlet operators on a path space with Gibbs measures via an SPDE approach

MHF2006-20 Masahisa TABATA

Energy stable finite element schemes and their applications to two-fluid flow problems

MHF2006-21 Yuzuru INAHAMA \& Hiroshi KAWABI

Asymptotic expansions for the Laplace approximations for Itô functionals of Brownian rough paths

MHF2006-22 Yoshiyuki KAGEI

Resolvent estimates for the linearized compressible Navier-Stokes equation in an infinite layer 
MHF2006-23 Yoshiyuki KAGEI

Asymptotic behavior of the semigroup associated with the linearized compressible Navier-Stokes equation in an infinite layer

MHF2006-24 Akihiro MIKODA, Shuichi INOKUCHI, Yoshihiro MIZOGUCHI \& Mitsuhiko FUJIO

The number of orbits of box-ball systems

MHF2006-25 Toru FUJII \& Sadanori KONISHI

Multi-class Logistic Discrimination via Wavelet-based Functionalization and Model Selection Criteria

MHF2006-26 Taro HAMAMOTO, Kenji KAJIWARA \& Nicholas S. WITTE

Hypergeometric Solutions to the $q$-Painlevé Equation of Type $\left(A_{1}+A_{1}^{\prime}\right)^{(1)}$

MHF2006-27 Hiroshi KAWABI \& Tomohiro MIYOKAWA

The Littlewood-Paley-Stein inequality for diffusion processes on general metric spaces

MHF2006-28 Hiroki MASUDA

Notes on estimating inverse-Gaussian and gamma subordinators under highfrequency sampling 